\title{
Generalizations of Weighted Version of Ostrowski's Inequality and Some Related Results
}

\author{
M. MATIĆ ${ }^{a, *}$, J. PEČARIĆ ${ }^{b, \dagger}$ and N. UJEVIĆc, \\ a FESB, Department of Mathematics, University of Split, R. Boškovića bb, \\ 21000 Split, Croatia; ${ }^{b}$ Faculty of Textile Technology, University of Zagreb, \\ Pierottijeva 6, 10000 Zagreb, Croatia; ${ }^{\mathrm{C}}$ Department of Mathematics, \\ University of Split, Teslina 12, 21000 Split, Croatia
}

(Received 13 July 1999; Revised 11 September 1999)

We establish some new weighted integral identities and use them to prove a number of inequalities of Ostrowski type. Among other results, we generalize one result related to the weighted version of the Ostrowski's inequality of Pečarić and Savić (Zbornik radova VA KoV (Beograd), 9 (1983), 171-202) as well as the recent result of Roumeliotis et al. (RGMIA, 1(1) (1998)). We also show that the recent Anastassiou's generalization (Proc. Amer. Math. Soc., 123 (1995), 3775-3781) of the Ostrowski's inequality is a special case of some results from this paper.

Keywords: Weighted integral identities; Ostrowski's inequality

1991 Mathematics Subject Classification: 26D10, 26D15

* Corresponding author. E-mail: mmatic@fesb.hr.

${ }^{\dagger}$ E-mail: jpecaric@maths.adelaide.edu.au.

${ }^{\ddagger}$ E-mail: ujevic@pmfst.hr. 


\section{INTRODUCTION}

The well-known Ostrowski's inequality is given by the following theorem [9]:

THEOREM 1 Let fbe a differentiable function on $[a, b]$ and let $\left|f^{\prime}(x)\right| \leq M$ on $[a, b]$. Then, for every $x \in[a, b]$,

$$
\left|f(x)-\frac{1}{b-a} \int_{a}^{b} f(t) \mathrm{d} t\right| \leq\left[\frac{1}{4}+\frac{(x-(a+b) / 2)^{2}}{(b-a)^{2}}\right](b-a) M .
$$

Some generalizations of this inequality, obtained by Milovanović $[5,6]$, Milovanović and Pečarić [7] and Fink [3], were noted in [8, pp. 468-471]. Recently, Anastassiou [1] proved some more general inequalities of this type. The basic result proved in [1] is the inequality

$$
\begin{aligned}
& \left|\frac{1}{b-a} \int_{a}^{b} f(t) \mathrm{d} t-f(x)\right| \\
& \leq \frac{1}{b-a} \sum_{j=1}^{n} \frac{\left|f^{(j)}(x)\right|}{(j+1) !}\left|(b-x)^{j+1}+(-1)^{j}(x-a)^{j+1}\right| \\
& \quad+\frac{\left\|f^{(n+1)}\right\|_{\infty}}{(n+2) !(b-a)}\left[(x-a)^{n+2}+(b-x)^{n+2}\right]
\end{aligned}
$$

which holds for any $x \in[a, b]$, whenever $f \in C^{n+1}([a, b]), n \in \mathbf{N}$. Under the additional assumption

$$
f^{(j)}(x)=0, \text { for all } j=1,2, \ldots, n,
$$

this inequality becomes

$\left|\frac{1}{b-a} \int_{a}^{b} f(t) \mathrm{d} t-f(x)\right| \leq \frac{\left\|f^{(n+1)}\right\|_{\infty}}{(n+2) !(b-a)}\left[(x-a)^{n+2}+(b-x)^{n+2}\right]$.

Further generalizations of the above results were deduced by Pearce and Pečarić [10]. Another possibility to generalize the inequality (1.1) is to consider the integral $\int_{a}^{b} f(t) w(t) \mathrm{d} t$, where $w$ is some weight function. 
More precisely, suppose $w:[a, b] \rightarrow[0, \infty)$ is integrable on the interval $I=[a, b]$ and

$$
\int_{I} w(t) \mathrm{d} t>0
$$

We call $w$ the weight function on the interval $I$. If $w$ is given, then we define the $j$ th moment $m_{j}(I ; w)$ of the interval $I$ with respect to $w$ as

$$
m_{j}(I ; w):=\int_{I} t^{j} w(t) \mathrm{d} t, \quad j=0,1,2, \ldots
$$

For any fixed $x \in I$, we define the $j$ th $x$-centered moment $E_{j}(x, I ; w)$ of the interval $I$ with respect to $w$ as

$$
E_{j}(x, I ; w):=\int_{I}(t-x)^{j} w(t) \mathrm{d} t, \quad j=0,1,2, \ldots
$$

Also, for any fixed $x \in I$ and for any real $r \in[0, \infty)$, we define the $r$ th $x$-centered absolute moment $M_{r}(x, I ; w)$ of the interval $I$ with respect to $w$ as

$$
M_{r}(x, I ; w):=\int_{I}|t-x|^{r} w(t) \mathrm{d} t .
$$

Note that $m_{0}(I ; w)=E_{0}(x, I ; w)=M_{0}(x, I ; w)=\int_{I} w(t) \mathrm{d} t$. Further, we define the mean $\mu(I ; w)$ and the variance $\sigma^{2}(I ; w)$ of the interval $I$ with respect to $w$ as

$$
\mu(I ; w):=\frac{m_{1}(I ; w)}{m_{0}(I ; w)} \quad \text { and } \quad \sigma^{2}(I ; w)=\frac{m_{2}(I ; w)}{m_{0}(I ; w)}-\mu^{2}(I ; w) .
$$

In the special case when $w(t)=1$ for all $t \in I=[a, b]$, we shall use the notation

$$
m_{j}:=m_{j}(I ; 1), \quad E_{j}(x):=E_{j}(x, I ; 1), \quad j=0,1,2, \ldots
$$

and

$$
M_{r}(x):=M_{r}(x, I ; 1), \quad r \in[0, \infty)
$$


A simple calculation gives

$$
m_{j}=\int_{a}^{b} t^{j} \mathrm{~d} t=\frac{b^{j+1}-a^{j+1}}{j+1}
$$

and

$$
E_{j}(x)=\int_{a}^{b}(t-x)^{j} \mathrm{~d} t=\frac{(b-x)^{j+1}+(-1)^{j}(x-a)^{j+1}}{j+1},
$$

for each $j=0,1,2, \ldots$ Also, for $r \in[0, \infty)$ we have

$$
\begin{aligned}
M_{r}(x) & =\int_{a}^{b}|t-x|^{r} \mathrm{~d} t \\
& =\int_{a}^{x}(x-t)^{r} \mathrm{~d} t+\int_{x}^{b}(t-x)^{r} \mathrm{~d} t \\
& =\frac{(x-a)^{r+1}+(b-x)^{r+1}}{r+1} .
\end{aligned}
$$

Further,

$$
\mu:=\mu(I ; 1)=\frac{a+b}{2} \quad \text { and } \quad \sigma^{2}:=\sigma^{2}(I ; 1)=\frac{(b-a)^{2}}{12} .
$$

Note that in (1.4)-(1.6) the weight function $w$ need not be bounded on $I$, and the interval $I=[a, b]$ may be replaced by any interval $I \subset \mathbf{R}$ (bounded or unbounded) - the quantities $m_{j}(I ; w), E_{j}(x, I ; w)$ and $M_{r}(x, I ; w)$ remain well defined, provided that the respective integrals converge. It is obvious that

$$
\begin{array}{rlrl}
\left|E_{j}(x, I ; w)\right| \leq M_{j}(x, I ; w) & & \text { for all } j ; \\
E_{j}(x, I ; w) & =M_{j}(x, I ; w) & & \text { for all even } j
\end{array}
$$

and, by the binomial formula,

$$
E_{k}(x, I ; w)=\sum_{j=0}^{k}\left(\begin{array}{l}
k \\
j
\end{array}\right)(-1)^{j} x^{j} m_{k-j}(I ; w), \quad k=1,2,3, \ldots
$$


The following theorem was proved in [12]:

THEOREM 2 Let $f, w:(a, b) \rightarrow \mathbf{R}$ be two mappings on $(a, b)$ with the following properties:

(1) $\sup \left|f^{\prime \prime}(t)\right|<\infty$,

(2) $w(t) \geq 0, \forall t \in(a, b)$,

(3) $\int_{a}^{b} w(t) \mathrm{d} t<\infty$.

Then for all $x \in(a, b)$ the following inequalities hold:

$$
\begin{aligned}
& \left|\frac{1}{m_{0}((a, b) ; w)} \int_{a}^{b} w(t) f(t) \mathrm{d} t-f(x)+[x-\mu((a, b) ; w)] f^{\prime}(x)\right| \\
& \leq \frac{\left\|f^{\prime \prime}\right\|_{\infty}}{2}\left\{[x-\mu((a, b) ; w)]^{2}+\sigma^{2}((a, b) ; w)\right\} \\
& \quad \leq \frac{\left\|f^{\prime \prime}\right\|_{\infty}}{2}\left(\left|x-\frac{a+b}{2}\right|+\frac{b-a}{2}\right)^{2} .
\end{aligned}
$$

The weighted version of the inequality (1.1) has been considered by Pečarić and Savić [11]. In [11, Teorema 8, p. 190] the following generalization of (1.1) was proved:

THEOREM 3 Let $w:[a, b] \rightarrow \mathbf{R}$ be a weight function on $[a, b]$. Suppose $f:[a, b] \rightarrow \mathbf{R}$ satisfies

$$
|f(t)-f(s)| \leq N|t-s|^{\alpha}, \text { for all } t, s \in[a, b]
$$

where $N>0$ and $0<\alpha \leq 1$ are some constants. Then for any $x \in[a, b]$ we have

$$
|f(x)-A(f ; w)| \leq N \frac{\int_{a}^{b}|t-x|^{\alpha} w(t) \mathrm{d} t}{\int_{a}^{b} w(t) \mathrm{d} t},
$$

where $A(f ; w):=\int_{a}^{b} f(t) w(t) \mathrm{d} t / \int_{a}^{b} w(t) \mathrm{d} t$. Further, iffor some constants $c$ and $\lambda$

$$
0<c \leq w(t) \leq \lambda c, \text { for all } t \in[a, b],
$$

then for any $x \in[a, b]$ we have

$$
|f(x)-A(f ; w)| \leq N \frac{\lambda L(x) J(x)}{L(x)-J(x)+\lambda J(x)}
$$


where

$$
\begin{aligned}
& L(x):=[\max \{x-a, b-x\}]^{\alpha} \text { and } \\
& J(x):=\frac{(x-a)^{1+\alpha}+(b-x)^{1+\alpha}}{(1+\alpha)(b-a)} .
\end{aligned}
$$

For $\alpha=1$ the condition (1.11) reduces to

$$
|f(t)-f(s)| \leq N|t-s|, \quad \text { for all } t, s \in[a, b] .
$$

This means that $f$ is $N$-Lipschitzian on $[a, b]$ and (1.13) holds with

$$
\begin{aligned}
& L(x):=\max \{x-a, b-x\} \quad \text { and } \\
& J(x):=\left[\frac{1}{4}+\frac{(x-(a+b) / 2)^{2}}{(b-a)^{2}}\right](b-a) .
\end{aligned}
$$

Moreover, for weight $w(t)=1, t \in[a, b]$ we can take $\lambda=1$ and (1.13) becomes

$$
\left|f(x)-\frac{1}{b-a} \int_{a}^{b} f(t) \mathrm{d} t\right| \leq N\left[\frac{1}{4}+\frac{(x-(a+b) / 2)^{2}}{(b-a)^{2}}\right](b-a) .
$$

This is, in fact, the Ostrowski's inequality for $N$-Lipschitzian function $f$ on $[a, b]$. As Pečarić and Savić noted in [11], the inequality (1.13) for $\alpha=1$ under assumption that $f$ is differentiable on $[a, b]$ and $\left|f^{\prime}(t)\right| \leq N$ for all $t \in[a, b]$ was proved by Milovanović [6, Teorema 1, pp. 24-26]. Also, note that the inequality (1.12) was rediscovered in [2, Theorem 2.1].

The proof of the inequality (1.13) is based on the following result of Karamata [4]:

THEOREM 4 Let $g, w:[a, b] \rightarrow \mathbf{R}$ be integrable on $[a, b]$. Suppose

$$
m \leq g(t) \leq M \quad \text { and } \quad 0<c \leq w(t) \leq \lambda c \quad(t \in[a, b])
$$

for some constants $m, M, c$ and $\lambda$. If $G$ and $A(g, w)$ are defined as

$$
G:=\frac{1}{b-a} \int_{a}^{b} g(t) \mathrm{d} t \quad \text { and } \quad A(g, w):=\frac{\int_{a}^{b} g(t) w(t) \mathrm{d} t}{\int_{a}^{b} w(t) \mathrm{d} t}
$$


then

$$
\begin{aligned}
\frac{\lambda m(M-G)+M(G-m)}{\lambda(M-G)+(G-m)} & \leq A(g, w) \\
& \leq \frac{m(M-G)+\lambda M(G-m)}{(M-G)+\lambda(G-m)} .
\end{aligned}
$$

The aim of this paper is to generalize the results stated in Theorems 2 and 3, as well as the inequalities (1.2) and (1.3). In Section 2 we establish some weighted integral identities. In Section 3 we use these identities to prove a number of new Ostrowski type inequalities. Moreover, we show that some of our results generalize Theorems 2 and 3. Also, we show that the inequalities (1.2) and (1.3) can be obtained as a special (nonweighted) case from some of our results.

\section{SOME INTEGRAL IDENTITIES}

The integral identities which we prove in this section are suitable to prove results of Section 3.

For given $a, b \in \mathbf{R}, a<b$ set $I=[a, b]$. Suppose $w: I \rightarrow[0, \infty)$ is a fixed weight function and define the kernel functions $K_{j}(\cdot, \cdot ; w): I^{2} \rightarrow \mathbf{R}$, $j \in \mathbf{N}$ by

$$
K_{j}(x, t ; w):= \begin{cases}\frac{1}{(j-1) !} \int_{a}^{t}(t-u)^{j-1} w(u) \mathrm{d} u, & \text { for } a \leq t<x \\ 0, & \text { for } t=x, \\ \frac{1}{(j-1) !} \int_{b}^{t}(t-u)^{j-1} w(u) \mathrm{d} u, & \text { for } x<t \leq b\end{cases}
$$

for all $x, t \in I$. Also, set

$$
K_{0}(x, t ; w):=w(t), \text { for all } x, t \in I .
$$

For fixed $x \in I$ and $j \in \mathbf{N}$, the function $K_{j}(x, \cdot ; w): I \rightarrow \mathbf{R}$ is continuous on $I \backslash\{x\}$ and

$$
\begin{aligned}
& K_{j}(x, a+0 ; w)=0 \text { for } a<x \leq b, \quad K_{j}(a, b-0 ; w)=0 \\
& K_{j}(x, b-0 ; w)=0 \text { for } a \leq x<b, \quad K_{j}(b, a+0 ; w)=0
\end{aligned}
$$


At the point of discontinuity $x, a<x<b$ we have

$$
\begin{aligned}
K_{j}(x, x-0 ; w) & =\lim _{t \rightarrow x-0} K_{j}(x, t ; w) \\
& =\frac{1}{(j-1) !} \int_{a}^{x}(x-u)^{j-1} w(u) \mathrm{d} u
\end{aligned}
$$

and

$$
\begin{aligned}
K_{j}(x, x+0 ; w) & =\lim _{t \rightarrow x+0} K_{j}(x, t ; w) \\
& =\frac{1}{(j-1) !} \int_{b}^{x}(x-u)^{j-1} w(u) \mathrm{d} u
\end{aligned}
$$

so that

$$
\begin{aligned}
K_{j}(x, x-0 ; w)-K_{j}(x, x+0 ; w) & =\frac{1}{(j-1) !} \int_{a}^{b}(x-u)^{j-1} w(u) \mathrm{d} u \\
& =\frac{(-1)^{j-1}}{(j-1) !} E_{j-1}(x, I ; w) .
\end{aligned}
$$

However, if we assume that $K_{j}(a, a-0 ; w)=0$ and $K_{j}(b, b+0 ; w)=0$, then (2.2) is also valid for $x=a$ and for $x=b$. If $a<t<x$, then

$$
\begin{aligned}
\frac{\mathrm{d}}{\mathrm{d} t} K_{j+1}(x, t ; w) & =\frac{\mathrm{d}}{\mathrm{d} t}\left[\frac{1}{j !} \int_{a}^{t}(t-u)^{j} w(u) \mathrm{d} u\right] \\
& =\frac{1}{j !}(t-t)^{j} w(t)+\frac{1}{j !} \int_{a}^{t} \frac{\partial}{\partial t}\left[(t-u)^{j} w(u)\right] \mathrm{d} u \\
& =\frac{1}{(j-1) !} \int_{a}^{t}(t-u)^{j-1} w(u) \mathrm{d} u \\
& =K_{j}(x, t ; w) .
\end{aligned}
$$

For $x<t<b$, similar calculation gives the same equality

$$
\frac{\mathrm{d}}{\mathrm{d} t} K_{j+1}(x, t ; w)=K_{j}(x, t ; w) .
$$


Therefore, (2.3) holds for $t \in(a, x) \cup(x, b)$ and for any $j \in \mathbf{N}$. It is easy to check that (2.3) holds for $t \in(a, x) \cup(x, b)$ and for $j=0$, as well. Using (2.1)-(2.3), for $j=0,1,2, \ldots$ and $a<x<b$, we get

$$
\begin{aligned}
\int_{a}^{b} K_{j}(x, t ; w) \mathrm{d} t= & \int_{a}^{x} K_{j}(x, t ; w) \mathrm{d} t+\int_{x}^{b} K_{j}(x, t ; w) \mathrm{d} t \\
= & \left.K_{j+1}(x, t ; w)\right|_{a} ^{x}+\left.K_{j+1}(x, t ; w)\right|_{x} ^{b} \\
= & K_{j+1}(x, x-0 ; w)-K_{j+1}(x, a+0 ; w) \\
& +K_{j+1}(x, b-0 ; w)-K_{j+1}(x, x+0 ; w) \\
= & K_{j+1}(x, x-0 ; w)-K_{j+1}(x, x+0 ; w) \\
= & \frac{(-1)^{j}}{j !} E_{j}(x, I ; w) .
\end{aligned}
$$

Also,

$$
\begin{aligned}
\int_{a}^{b} K_{j}(a, t ; w) \mathrm{d} t & =\left.K_{j+1}(a, t ; w)\right|_{a} ^{b} \\
& =K_{j+1}(a, b-0 ; w)-K_{j+1}(a, a+0 ; w) \\
& =-K_{j+1}(a, a+0 ; w) \\
& =\frac{(-1)^{j}}{j !} E_{j}(a, I ; w)
\end{aligned}
$$

and

$$
\begin{aligned}
\int_{a}^{b} K_{j}(b, t ; w) \mathrm{d} t & =\left.K_{j+1}(b, t ; w)\right|_{a} ^{b} \\
& =K_{j+1}(b, b-0 ; w)-K_{j+1}(b, a+0 ; w) \\
& =K_{j+1}(b, b-0 ; w) \\
& =\frac{(-1)^{j}}{j !} E_{j}(b, I ; w) .
\end{aligned}
$$

Therefore,

$$
\int_{a}^{b} K_{j}(x, t ; w) \mathrm{d} t=\frac{(-1)^{j}}{j !} E_{j}(x, I ; w)
$$


holds for $j=0,1,2, \ldots$ and $x \in I$. Finally, when $w(t)=1$ for all $t \in I$, we define $K_{j}(\cdot, \cdot): I^{2} \rightarrow \mathbf{R}$ as

$$
K_{j}(x, t):=K_{j}(x, t ; 1), \quad x, t \in I, j=0,1,2, \ldots
$$

Thus, $K_{0}(x, t)=1$ for all $x, t \in I$ and for $j \in \mathbf{N}$ we have

$$
K_{j}(x, t):= \begin{cases}\frac{(t-a)^{j}}{j !}, & \text { for } a \leq t<x, \\ 0, & \text { for } t=x, \\ \frac{(t-b)^{j}}{j !}, & \text { for } x<t \leq b .\end{cases}
$$

The following theorem is the key result of our paper.

THEOREM 5 Let function $f: J \rightarrow \mathbf{R}$ be defined on the interval $J \subset \mathbf{R}$. For $a, b \in J, a<b$ set $I=[a, b]$. For $j=0,1,2, \ldots$ let $K_{j}(x, t ; w)$ be defined as above. Suppose that, for some $n \in \mathbf{N} \cup\{0\}, f^{(n)}(t)$ exists for all $t \in I$, with usual convention $f^{(0)}(t)=f(t)$. Set

$$
\mathcal{R}_{n}(x, f ; w):=\int_{a}^{b} f(t) w(t) \mathrm{d} t-\sum_{j=0}^{n} \frac{f^{(j)}(x)}{j !} E_{j}(x, I ; w), \quad x \in I .
$$

If $f^{(n)}(\cdot)$ is integrable on $I$, then $R_{n}(x, f ; w)$ is well defined for all $x \in I$ and

$$
\mathcal{R}_{n}(x, f ; w)=(-1)^{n} \int_{a}^{b}\left[f^{(n)}(t)-f^{(n)}(x)\right] K_{n}(x, t ; w) \mathrm{d} t .
$$

Further, if $f^{(n)}(\cdot)$ is continuous function of bounded variation on I, then for all $x \in I$

$$
\mathcal{R}_{n}(x, f ; w)=(-1)^{n+1} \int_{a}^{b} K_{n+1}(x, t ; w) \mathrm{d} f^{(n)}(t) .
$$

Especially, when $f^{(n+1)}(t)$ exists for all $t \in I$, if $f^{(n+1)}(\cdot)$ is integrable on $I$, then for all $x \in I$

$$
\mathcal{R}_{n}(x, f ; w)=(-1)^{n+1} \int_{a}^{b} K_{n+1}(x, t ; w) f^{(n+1)}(t) \mathrm{d} t .
$$

(The integrals in (2.5) and (2.7) are ordinary Riemann integrals, while in (2.6) we have the Riemann-Stieltjes integral.) 
Proof For $j=0,1, \ldots, n$ denote

$$
D_{j}:=(-1)^{j} \int_{a}^{b}\left[f^{(j)}(t)-f^{(j)}(x)\right] K_{j}(x, t ; w) \mathrm{d} t .
$$

By (2.4), we have

$$
\begin{aligned}
D_{j} & =(-1)^{j} \int_{a}^{b} f^{(j)}(t) K_{j}(x, t ; w) \mathrm{d} t-(-1)^{j} f^{(j)}(x) \int_{a}^{b} K_{j}(x, t ; w) \mathrm{d} t \\
& =(-1)^{j} \int_{a}^{b} f^{(j)}(t) K_{j}(x, t ; w) \mathrm{d} t-\frac{f^{(j)}(x)}{j !} E_{j}(x, I ; w) .
\end{aligned}
$$

If $n=0$, then

$$
\begin{aligned}
D_{0} & =\int_{a}^{b} f(t) K_{0}(x, t ; w) \mathrm{d} t-f(x) E_{0}(x, I ; w) \\
& =\int_{a}^{b} f(t) w(t) \mathrm{d} t-f(x) E_{0}(x, I ; w) \\
& =\mathcal{R}_{0}(x, f ; w)
\end{aligned}
$$

which shows that (2.5) is valid for $n=0$. If $n \geq 1$, then for any $j=$ $0,1, \ldots, n-1$ by partial integration and using $(2.2)-(2.4)$, we have

$$
\begin{aligned}
\int_{a}^{b} & f^{(j+1)}(t) K_{j+1}(x, t ; w) \mathrm{d} t \\
= & \int_{a}^{x} f^{(j+1)}(t) K_{j+1}(x, t ; w) \mathrm{d} t+\int_{x}^{b} f^{(j+1)}(t) K_{j+1}(x, t ; w) \mathrm{d} t \\
= & \left.f^{(j)}(t) K_{j+1}(x, t ; w)\right|_{a} ^{x}-\int_{a}^{x} f^{(j)}(t) K_{j}(x, t ; w) \mathrm{d} t \\
& +\left.f^{(j)}(t) K_{j+1}(x, t ; w)\right|_{x} ^{b}-\int_{x}^{b} f^{(j)}(t) K_{j}(x, t ; w) \mathrm{d} t \\
= & f^{(j)}(x)\left[K_{j+1}(x, x-0 ; w)-K_{j+1}(x, x+0 ; w)\right] \\
& -\int_{a}^{b} f^{(j)}(t) K_{j}(x, t ; w) \mathrm{d} t \\
= & (-1)^{j} \frac{f^{(j)}(x)}{j !} E_{j}(x, I ; w)-\int_{a}^{b} f^{(j)}(t) K_{j}(x, t ; w) \mathrm{d} t
\end{aligned}
$$


Here we assume that $\int_{a}^{x} f^{(j+1)}(t) K_{j+1}(x, t ; w) \mathrm{d} t=0$ for $x=a$, while $\int_{x}^{b} f^{(j+1)}(t) K_{j+1}(x, t ; w) \mathrm{d} t=0$ for $x=b$. Multiplying by $(-1)^{j+1}$ and using (2.8) we get

$$
\begin{aligned}
& (-1)^{j+1} \int_{a}^{b} f^{(j+1)}(t) K_{j+1}(x, t ; w) \mathrm{d} t \\
& =(-1)^{j} \int_{a}^{b} f^{(j)}(t) K_{j}(x, t ; w) \mathrm{d} t-\frac{f^{(j)}(x)}{j !} E_{j}(x, I ; w) \\
& =D_{j} .
\end{aligned}
$$

Combining this with (2.8) we get

$$
\begin{aligned}
D_{j+1} & =(-1)^{j+1} \int_{a}^{b} f^{(j+1)}(t) K_{j+1}(x, t ; w) \mathrm{d} t-\frac{f^{(j+1)}(x)}{(j+1) !} E_{j+1}(x, I ; w) \\
& =D_{j}-\frac{f^{(j+1)}(x)}{(j+1) !} E_{j+1}(x, I ; w), \quad j=0,1, \ldots, n-1 .
\end{aligned}
$$

From (2.10) and (2.9) it is easy to obtain

$$
\begin{aligned}
D_{n} & =\int_{a}^{b} f(t) w(t) \mathrm{d} t-\sum_{j=0}^{n} \frac{f^{(j)}(x)}{j !} E_{j}(x, I ; w) \\
& =\mathcal{R}_{n}(x, f ; w),
\end{aligned}
$$

which is just the equality (2.5). Let us now prove (2.6). First, by applying partial integration for the Riemann-Stieltjes integral to the right hand side of (2.6), we have

$$
\begin{aligned}
(-1)^{n+1} \int_{a}^{b} K_{n+1}(x, t ; w) \mathrm{d} f^{(n)}(t) \\
=(-1)^{n+1} \int_{a}^{b} K_{n+1}(x, t ; w) \mathrm{d}\left[f^{(n)}(t)-f^{(n)}(x)\right] \\
=\left.(-1)^{n+1}\left[f^{(n)}(t)-f^{(n)}(x)\right] K_{n+1}(x, t ; w)\right|_{a} ^{b} \\
\quad+(-1)^{n} \int_{a}^{b}\left[f^{(n)}(t)-f^{(n)}(x)\right] \mathrm{d} K_{n+1}(x, t ; w) \\
=(-1)^{n} \int_{a}^{b}\left[f^{(n)}(t)-f^{(n)}(x)\right] \mathrm{d} K_{n+1}(x, t ; w) .
\end{aligned}
$$


The kernel function $K_{n+1}(x, \cdot ; w)$ is continuous on $I \backslash\{x\}$, while at the point of discontinuity $x$ the value of the function $f^{(n)}(\cdot)-f^{(n)}(x)$ is zero. Since (2.3) holds, it is easy to check that

$\int_{a}^{b}\left[f^{(n)}(t)-f^{(n)}(x)\right] \mathrm{d} K_{n+1}(x, t ; w)=\int_{a}^{b}\left[f^{(n)}(t)-f^{(n)}(x)\right] K_{n}(x, t ; w) \mathrm{d} t$ holds for all $x \in I$. Now (2.6) follows from (2.5). Finally, (2.7) is a consequence of the fact that for all $x \in I$

$$
\int_{a}^{b} K_{n+1}(x, t ; w) \mathrm{d} f^{(n)}(t)=\int_{a}^{b} K_{n+1}(x, t ; w) f^{(n+1)}(t) \mathrm{d} t
$$

when $f^{(n+1)}(\cdot)$ exists and is integrable on $I$.

Remark 1 When $w(t)=1, t \in I$ we use the notation $\mathcal{R}_{n}(x, f)$ for $\mathcal{R}_{n}(x, f ; 1)$. By $(1.7)$ we have for all $x \in I$

$$
\begin{aligned}
\mathcal{R}_{n}(x, f) & =\int_{a}^{b} f(t) w(t) \mathrm{d} t-\sum_{j=0}^{n} \frac{f^{(j)}(x)}{j !} E_{j}(x) \\
& =\int_{a}^{b} f(t) \mathrm{d} t-\sum_{j=0}^{n} \frac{f^{(j)}(x)}{(j+1) !}\left[(b-x)^{j+1}+(-1)^{j}(x-a)^{j+1}\right] .
\end{aligned}
$$

The integral identities $(2.5)-(2.7)$ reduce to

$$
\begin{aligned}
& \mathcal{R}_{n}(x, f)=(-1)^{n} \int_{a}^{b}\left[f^{(n)}(t)-f^{(n)}(x)\right] K_{n}(x, t) \mathrm{d} t \\
& \mathcal{R}_{n}(x, f)=(-1)^{n+1} \int_{a}^{b} K_{n+1}(x, t) \mathrm{d} f^{(n)}(t),
\end{aligned}
$$

and

$$
\mathcal{R}_{n}(x, f)=(-1)^{n+1} \int_{a}^{b} K_{n+1}(x, t) f^{(n+1)}(t) \mathrm{d} t,
$$

respectively. The above equalities are valid for all $x \in I$, under appropriate assumptions on $f$. 


\section{SOME OSTROWSKI TYPE INEQUALITIES}

In this section we use integral identities (2.5)-(2.7) to prove some Ostrowski type inequalities. Let $f: J \rightarrow \mathbf{R}$ be a function defined on the interval $J \subset \mathbf{R}$ and let $w: I \rightarrow[0, \infty)$ be a weight function defined on $I=[a, b] \subset J$. Suppose that, for some $n \in \mathbf{N} \cup\{0\}, f^{(n)}(t)$ exists for all $t \in I$. For $x \in I$ define

$$
\mathcal{O}_{n}(x, f ; w):=\frac{\mathcal{R}_{n}(x, f ; w)}{m_{0}(I ; w)}, \quad \mathcal{O}_{n}(x, f):=\frac{\mathcal{R}_{n}(x, f)}{b-a}
$$

Note that for $n=0$ we have

$$
\begin{aligned}
\mathcal{O}_{0}(x, f ; w) & =\frac{\int_{a}^{b} f(t) w(t) \mathrm{d} t}{\int_{a}^{b} w(t) \mathrm{d} t}-f(x), \\
\mathcal{O}_{0}(x, f) & =\frac{1}{b-a} \int_{a}^{b} f(t) \mathrm{d} t-f(x), \quad x \in I,
\end{aligned}
$$

while for $n \geq 1$ we can write

$$
\mathcal{O}_{n}(x, f ; w)=\mathcal{O}_{0}(x, f ; w)-\frac{1}{m_{0}(I ; w)} \sum_{j=1}^{n} \frac{f^{(j)}(x)}{j !} E_{j}(x, I ; w)
$$

and

$\mathcal{O}_{n}(x, f)=\mathcal{O}_{0}(x, f)-\frac{1}{b-a} \sum_{j=1}^{n} \frac{f^{(j)}(x)}{(j+1) !}\left[(b-x)^{j+1}+(-1)^{j}(x-a)^{j+1}\right]$,

for all $x \in I$.

\subsection{Inequalities Involving $f^{(n)}$}

We first derive some inequalities which are obtained from the identity (2.6) and involve the total variation $V_{a}^{b}\left(f^{(n)}\right)$. 
THEOREM 6 Let $f^{(n)}(\cdot)$ be a continuous function of bounded variation on I. Then for all $x \in I$ we have

$$
\left|\mathcal{O}_{n}(x, f ; w)\right| \leq \frac{V_{a}^{b}\left(f^{(n)}\right)}{n ! m_{0}(I ; w)} \max \left\{\int_{a}^{x}(x-u)^{n} w(u) \mathrm{d} u, \int_{x}^{b}(u-x)^{n} w(u) \mathrm{d} u\right\},
$$

where $V_{a}^{b}\left(f^{(n)}\right)=\int_{a}^{b}\left|\mathrm{~d} f^{(n)}(t)\right|$ is the total variation of the function $f^{(n)}(\cdot)$ on I. Also, for all $x \in I$

$$
\left|\mathcal{O}_{n}(x, f)\right| \leq \frac{V_{a}^{b}\left(f^{(n)}\right)}{(n+1) !(b-a)}\left[\frac{b-a}{2}+\left|x-\frac{a+b}{2}\right|\right]^{n+1} .
$$

Proof From (2.6) we get the estimate

$$
\begin{aligned}
\left|\mathcal{R}_{n}(x, f ; w)\right| & \leq \int_{a}^{b}\left|K_{n+1}(x, t ; w)\right|\left|\mathrm{d} f^{(n)}(t)\right| \\
& \leq \sup _{a \leq t \leq b}\left|K_{n+1}(x, t ; w)\right| \int_{a}^{b}\left|\mathrm{~d} f^{(n)}(t)\right| \\
& =\sup _{a \leq t \leq b}\left|K_{n+1}(x, t ; w)\right| V_{a}^{b}\left(f^{(n)}\right) .
\end{aligned}
$$

For fixed $j \in \mathbf{N}$ and $x \in I$ we have

$$
\left|K_{j}(x, t ; w)\right|= \begin{cases}\frac{1}{(j-1) !} \int_{a}^{t}(t-u)^{j-1} w(u) \mathrm{d} u, & \text { for } a \leq t<x, \\ 0, & \text { for } t=x, \\ \frac{1}{(j-1) !} \int_{t}^{b}(u-t)^{j-1} w(u) \mathrm{d} u, & \text { for } x<t \leq b .\end{cases}
$$

It is obvious that

$$
\begin{aligned}
& \sup _{a \leq t \leq b}\left|K_{j}(x, t ; w)\right| \\
& \quad=\frac{1}{(j-1) !} \max \left\{\int_{a}^{x}(x-u)^{j-1} w(u) \mathrm{d} u, \int_{x}^{b}(u-x)^{j-1} w(u) \mathrm{d} u\right\} .
\end{aligned}
$$


Combining (3.5) for $j=n+1$ with (3.3) divided by $m_{0}(I ; w)$, we get (3.1). Further, if $w(t)=1, t \in I$, then

$$
\left|K_{j}(x, t)\right|= \begin{cases}\frac{(t-a)^{j}}{j !}, & \text { for } a \leq t<x \\ 0, & \text { for } t=x \\ \frac{(b-t)^{j}}{j !}, & \text { for } x<t \leq b\end{cases}
$$

and

$$
\begin{aligned}
\sup _{a \leq t \leq b}\left|K_{j}(x, t)\right| & =\frac{1}{j !} \max \left\{(x-a)^{j},(b-x)^{j}\right\} \\
& =\frac{1}{j !}[\max \{x-a, b-x\}]^{j} \\
& =\frac{1}{j !}\left[\frac{b-a}{2}+\left|x-\frac{a+b}{2}\right|\right]^{j} .
\end{aligned}
$$

Here we used the equality $\max \{A, B\}=\frac{1}{2}[A+B+|A-B|]$ which holds for any $A, B \in \mathbf{R}$. Combining (3.7) for $j=n+1$ with (3.3) for $w=1$, after dividing by $b-a$, we get (3.2).

Remark 2 For $n=0$, Theorem 6 gives the inequalities

$$
\left|\frac{\int_{a}^{b} f(t) w(t) \mathrm{d} t}{\int_{a}^{b} w(t) \mathrm{d} t}-f(x)\right| \leq \frac{V_{a}^{b}(f)}{\int_{a}^{b} w(t) \mathrm{d} t} \max \left\{\int_{a}^{x} w(u) \mathrm{d} u, \int_{x}^{b} w(u) \mathrm{d} u\right\}
$$

and

$$
\left|\frac{1}{b-a} \int_{a}^{b} f(t) \mathrm{d} t-f(x)\right| \leq \frac{V_{a}^{b}(f)}{b-a}\left[\frac{b-a}{2}+\left|x-\frac{a+b}{2}\right|\right]
$$

which are valid for all $x \in I$, provided $f(\cdot)$ is a continuous function of bounded variation on $I$.

Next, we make use of the integral identity (2.5) to deduce some further Ostrowski type inequalities. The simples inequalities, expressed in terms 
of the $L_{p}$-norms, $1 \leq p \leq \infty$, follow directly from (2.5). Namely, if $p, q$ is a pair of conjugate exponents, that is

$p=1, q=\infty \quad$ or $\quad p=\infty, q=1 \quad$ or $\quad 1<p, q<\infty, \frac{1}{p}+\frac{1}{q}=1$

then (2.5) implies

$$
\begin{aligned}
\left|\mathcal{O}_{n}(x, f ; w)\right| & =\frac{\left|\mathcal{R}_{n}(x, f ; w)\right|}{m_{0}(I ; w)} \\
& \leq \frac{1}{m_{0}(I ; w)} \int_{a}^{b}\left|f^{(n)}(t)-f^{(n)}(x)\right|\left|K_{n}(x, t ; w)\right| \mathrm{d} t \\
& \leq \frac{1}{m_{0}(I ; w)}\left\|f^{(n)}(\cdot)-f^{(n)}(x)\right\|_{p}\left\|K_{n}(x, ; w w)\right\|_{q} .
\end{aligned}
$$

Recall that for a function $g(\cdot)$ defined and integrable on $I=[a, b]$ we have

$$
\begin{aligned}
\|g(\cdot)\|_{\infty} & :=\sup _{a \leq t \leq b}|g(t)| \text { and } \\
\|g(\cdot)\|_{r}: & =\left(\int_{a}^{b}|g(t)|^{r}\right)^{1 / r}, \quad 1 \leq r<\infty .
\end{aligned}
$$

When $w(t)=1, t \in I$ we can calculate $\left\|K_{n}(x, \cdot)\right\|_{q}$. By (3.7) we have

$$
\left\|K_{n}(x, \cdot)\right\|_{\infty}=\frac{1}{n !}\left[\frac{b-a}{2}+\left|x-\frac{a+b}{2}\right|\right]^{n}, \quad n \in \mathbf{N} \cup\{0\}, x \in I
$$

For $1 \leq q<\infty$, using (3.6) we have

$$
\begin{aligned}
\int_{a}^{b}\left|K_{n}(x, t)\right|^{q} \mathrm{~d} t & =\frac{1}{(n !)^{q}} \int_{a}^{x}(t-a)^{q n} \mathrm{~d} t+\frac{1}{(n !)^{q}} \int_{x}^{b}(b-t)^{q n} \mathrm{~d} t \\
& =\frac{(x-a)^{q n+1}+(b-x)^{q n+1}}{(n !)^{q}(q n+1)}
\end{aligned}
$$


so that

$\left\|K_{n}(x, \cdot)\right\|_{q}=\frac{1}{n !}\left[\frac{(x-a)^{q n+1}+(b-x)^{q n+1}}{q n+1}\right]^{1 / q}, \quad n \in \mathbf{N} \cup\{0\}, x \in I$.

In the case of general weight function $w$, it should be noted that $K_{n}(x, t ; w) \geq 0$ for $t \in[a, x)$ and for all $n \in \mathbf{N} \cup\{0\}$, while for $t \in(x, b]$ we have $K_{n}(x, t ; w) \geq 0$ when $n$ is even and $K_{n}(x, t ; w) \leq 0$ when $n$ is odd. So, we can write

$$
\left|K_{n}(x, t ; w)\right|= \begin{cases}K_{n}(x, t ; w), & \text { for } a \leq t<x \\ (-1)^{n} K_{n}(x, t ; w), & \text { for } x<t \leq b\end{cases}
$$

Using (2.1) and (2.3), for $a<x<b$ we get

$$
\begin{aligned}
\int_{a}^{b}\left|K_{n}(x, t ; w)\right| \mathrm{d} t & =\int_{a}^{x} K_{n}(x, t ; w) \mathrm{d} t+(-1)^{n} \int_{x}^{b} K_{n}(x, t ; w) \mathrm{d} t \\
& =\left.K_{n+1}(x, t ; w)\right|_{a} ^{x}+\left.(-1)^{n} K_{n+1}(x, t ; w)\right|_{x} ^{b} \\
& =K_{n+1}(x, x-0 ; w)-(-1)^{n} K_{n+1}(x, x+0 ; w) \\
& =\frac{1}{n !} \int_{a}^{x}(x-u)^{n} w(u) \mathrm{d} u+\frac{1}{n !} \int_{x}^{b}(u-x)^{n} w(u) \mathrm{d} u \\
& =\frac{1}{n !} M_{n}(x, I ; w) .
\end{aligned}
$$

It is easy to see that the similar calculation is valid for $x=a$ and for $x=b$, as well. Therefore,

$$
\left\|K_{n}(x, \cdot ; w)\right\|_{1}=\frac{M_{n}(x, I ; w)}{n !}, \quad n \in \mathbf{N} \cup\{0\}, x \in I .
$$

THEOREM 7 Assume that, for some $n \in \mathbf{N} \cup\{0\}, f^{(n)}(t)$ exists for all $t \in I$, and that, $f^{(n)}(\cdot)$ is integrable on I. Then for all $x \in I$ we have

$$
\left|\mathcal{O}_{n}(x, f ; w)\right| \leq \frac{M_{n}(x, I ; w)}{n ! m_{0}(I ; w)}\left\|f^{(n)}(\cdot)-f^{(n)}(x)\right\|_{\infty}
$$


and

$$
\left|\mathcal{O}_{n}(x, f)\right| \leq \frac{(x-a)^{n+1}+(b-x)^{n+1}}{(n+1) !(b-a)}\left\|f^{(n)}(\cdot)-f^{(n)}(x)\right\|_{\infty}
$$

Also

$$
\left|\mathcal{O}_{n}(x, f ; w)\right| \leq \frac{\left\|K_{n}(x, \cdot ; w)\right\|_{\infty}}{m_{0}(I ; w)} \int_{a}^{b}\left|f^{(n)}(t)-f^{(n)}(x)\right| \mathrm{d} t
$$

and

$$
\left|\mathcal{O}_{n}(x, f)\right| \leq \frac{[(b-a) / 2+|x-(a+b) / 2|]^{n}}{n !(b-a)} \int_{a}^{b}\left|f^{(n)}(t)-f^{(n)}(x)\right| \mathrm{d} t
$$

Finally, if $1<p, q<\infty$ and $(1 / p)+(1 / q)=1$, then for all $x \in I$ we have

$$
\left|\mathcal{O}_{n}(x, f ; w)\right| \leq \frac{1}{m_{0}(I ; w)}\left\|f^{(n)}(\cdot)-f^{(n)}(x)\right\|_{p}\left\|K_{n}(x, \cdot ; w)\right\|_{q}
$$

and

$$
\left|\mathcal{O}_{n}(x, f)\right| \leq \frac{1}{n !}\left[\frac{(x-a)^{q n+1}+(b-x)^{q n+1}}{q n+1}\right]^{1 / q}\left\|f^{(n)}(\cdot)-f^{(n)}(x)\right\|_{p}
$$

Proof Set $p=\infty$ and $q=1$ in (3.9). Then (3.13) follows from (3.12), while (3.14) follows from (3.11) with $q=1$. Similarly, (3.15) and (3.16) follow from (3.9) with $p=1$ and $q=\infty$ (here we additionally use (3.10) to obtain (3.16)). Finally, if $1<p, q<\infty$, then (3.17) coincides with (3.9), while (3.18) follows from (3.11).

Another way to use the identity (2.5) is to put additional assumption of $f^{(n)}(\cdot)$. 
DEFINITION 1 Let $N>0$ and $\alpha>0$. We say that function $g: I \rightarrow \mathbf{R}$ is of class $C_{\alpha, N}(I)$ if

$$
|g(t)-g(s)| \leq N|t-s|^{\alpha}, \text { for all } t, s \in I .
$$

If $g$ is of class $C_{1, N}(I)$, that is if

$$
|g(t)-g(s)| \leq N|t-s|, \quad \text { for all } t, s \in I,
$$

then we say that $g$ is $N$-Lipschitzian on $I$.

If we assume that $f^{(n)}(\cdot)$ is of class $C_{\alpha, N}(I)$ for some constants $N>0$ and $\alpha>0$, then from (2.5) we get the estimate

$$
\begin{aligned}
\left|\mathcal{O}_{n}(x, f ; w)\right| & =\frac{\left|\mathcal{R}_{n}(x, f ; w)\right|}{m_{0}(I ; w)} \\
& \leq \frac{1}{m_{0}(I ; w)} \int_{a}^{b}\left|f^{(n)}(t)-f^{(n)}(x)\right|\left|K_{n}(x, t ; w)\right| \mathrm{d} t \\
& \leq \frac{N}{m_{0}(I ; w)} \int_{a}^{b}|t-x|^{\alpha}\left|K_{n}(x, t ; w)\right| \mathrm{d} t .
\end{aligned}
$$

Using (3.4), for $n \in \mathbf{N}$ we get

$$
\begin{aligned}
\int_{a}^{b}|t-x|^{\alpha}\left|K_{n}(x, t ; w)\right| \mathrm{d} t \\
=\frac{1}{(n-1) !} \int_{a}^{x}(x-t)^{\alpha} \int_{a}^{t}(t-u)^{n-1} w(u) \mathrm{d} u \mathrm{~d} t \\
\quad+\frac{1}{(n-1) !} \int_{x}^{b}(t-x)^{\alpha} \int_{a}^{t}(u-t)^{n-1} w(u) \mathrm{d} u \mathrm{~d} t \\
=\frac{1}{(n-1) !} \int_{a}^{x} w(u) \int_{u}^{x}(x-t)^{\alpha}(t-u)^{n-1} \mathrm{~d} t \mathrm{~d} u \\
\quad+\frac{1}{(n-1) !} \int_{x}^{b} w(u) \int_{x}^{u}(t-x)^{\alpha}(u-t)^{n-1} \mathrm{~d} t \mathrm{~d} u .
\end{aligned}
$$

Further, by substituting $t=u+(x-u) s$, we get

$$
\int_{u}^{x}(x-t)^{\alpha}(t-u)^{n-1} \mathrm{~d} t=(x-u)^{\alpha+n} \int_{0}^{1}(1-s)^{\alpha} s^{n-1} \mathrm{~d} s, \quad \text { for } a<u<x
$$


and

$\int_{x}^{u}(t-x)^{\alpha}(u-t)^{n-1} \mathrm{~d} t=(u-x)^{\alpha+n} \int_{0}^{1}(1-s)^{\alpha} s^{n-1} \mathrm{~d} s$, for $x<u<b$

Therefore,

$$
\begin{aligned}
& \int_{a}^{b}|t-x|^{\alpha}\left|K_{n}(x, t ; w)\right| \mathrm{d} t \\
& \quad=\frac{\int_{0}^{1}(1-s)^{\alpha} s^{n-1} \mathrm{~d} s}{(n-1) !}\left[\int_{a}^{x}(x-u)^{\alpha+n} w(u) \mathrm{d} u+\int_{x}^{b}(u-x)^{\alpha+n} w(u) \mathrm{d} u\right] \\
& \quad=\frac{B(\alpha+1, n)}{(n-1) !} \int_{a}^{b}|u-x|^{\alpha+n} w(u) \mathrm{d} u \\
& =\frac{B(\alpha+1, n)}{(n-1) !} M_{\alpha+n}(x, I: w)
\end{aligned}
$$

where

$$
B(x, y)=\int_{0}^{1}(1-s)^{x-1} s^{y-1} \mathrm{~d} s \quad(x>0, y>0)
$$

is the Beta function. Since $B(x, y)=\Gamma(x) \Gamma(y) / \Gamma(x+y)$, where $\Gamma$ is the Gamma function, and since $\Gamma(z+1)=z \Gamma(z), z>0$ and $\Gamma(k)=(k-1)$ !, $k \in \mathbf{N}$, we have

$$
\begin{aligned}
\frac{B(\alpha+1, n)}{(n-1) !} & =\frac{\Gamma(\alpha+1) \Gamma(n)}{(n-1) ! \Gamma(\alpha+n+1)}=\frac{\Gamma(\alpha+1)}{\Gamma(\alpha+n+1)} \\
& =\frac{1}{(\alpha+1)(\alpha+2) \cdots(\alpha+n)}
\end{aligned}
$$

Now, from (3.19) and (3.20) we get

$$
\left|\mathcal{O}_{n}(x, f ; w)\right| \leq N \frac{\Gamma(\alpha+1) M_{\alpha+n}(x, I: w)}{\Gamma(\alpha+n+1) m_{0}(I ; w)}
$$


for any $x \in I$ and $n \in \mathbf{N}$. For $n=0$, (3.19) gives

$$
\begin{aligned}
\left|\mathcal{O}_{0}(x, f ; w)\right| & \leq \frac{N}{m_{0}(x, I ; w)} \int_{a}^{b}|t-x|^{\alpha} w(t) \mathrm{d} t \\
& =N \frac{M_{\alpha}(x, I ; w)}{m_{0}(I ; w)} .
\end{aligned}
$$

This shows that (3.20) is valid for $n=0$, as well, since for $n=0$ obviously $\Gamma(\alpha+n+1)=\Gamma(\alpha+1)$. Now we can state our next theorem.

THEOREM 8 Assume that, for some $n \in \mathbf{N} \cup\{0\}, f^{(n)}(t)$ exists for all $t \in I$. Suppose that $f^{(n)}(\cdot)$ is of class $C_{\alpha, N}(I)$ for some constants $N>0$ and $\alpha>0$. Then, for all $x \in I$ we have

$$
\left|\mathcal{O}_{n}(x, f ; w)\right| \leq N \frac{\Gamma(\alpha+1) M_{\alpha+n}(x, I: w)}{\Gamma(\alpha+n+1) m_{0}(I ; w)}
$$

and

$$
\left|\mathcal{O}_{n}(x, f)\right| \leq N \frac{\Gamma(\alpha+1)\left[(x-a)^{\alpha+n+1}+(b-x)^{\alpha+n+1}\right]}{\Gamma(\alpha+n+2)(b-a)} .
$$

If

$$
0<c \leq w(t) \leq \lambda c, \quad t \in I
$$

for some constants $c$ and $\lambda$, then for all $x \in I$ we have

$$
\left|\mathcal{O}_{n}(x, f ; w)\right| \leq N \frac{\Gamma(\alpha+1) \lambda L_{\alpha+n}(x) J_{\alpha+n+1}(x)}{\Gamma(\alpha+n+1)\left[L_{\alpha+n}(x)-J_{\alpha+n+1}(x)+\lambda J_{\alpha+n+1}(x)\right]},
$$

where

$$
\begin{aligned}
& L_{s}(x)=\left[\frac{b-a}{2}+\left|x-\frac{a+b}{2}\right|\right]^{s}, \\
& J_{s}(x)=\frac{(x-a)^{s}+(b-x)^{s}}{s(b-a)}, \quad s>0 .
\end{aligned}
$$


Proof The first estimate coincides with (3.21) and it is already proved. The estimate (3.22) is the special case of (3.21) and follows from the equality (1.8) for $r=\alpha+n$. Further, note that

$$
0 \leq|u-x|^{\alpha+n} \leq \max \left\{(x-a)^{\alpha+n},(b-x)^{\alpha+n}\right\} \quad \text { for all } u \in I .
$$

By the similar calculation as used in proving (3.7), we have $\max \left\{(x-a)^{\alpha+n},(b-x)^{\alpha+n}\right\}=L_{\alpha+n}(x)$. If we set $g(u)=|u-x|^{\alpha+n}, u \in I$, then (1.8) with $r=\alpha+n$ gives

$$
G=\frac{1}{b-a} \int_{a}^{b}|u-x|^{\alpha+n} \mathrm{~d} u=\frac{M_{\alpha+n}(x)}{b-a}=J_{\alpha+n+1}(x) .
$$

Now we apply the right hand side inequality of (1.14) for $m=0$, and $M=L_{\alpha+n}(x)$ to obtain

$$
\frac{M_{\alpha+n}(x, I: w)}{m_{0}(I ; w)} \leq \frac{\lambda L_{\alpha+n}(x) J_{\alpha+n+1}(x)}{L_{\alpha+n}(x)-J_{\alpha+n+1}(x)+\lambda J_{\alpha+n+1}(x)} .
$$

Now (3.23) follows from (3.21).

Remark 3 From the proof of Theorem 8, we see that the following simple estimate holds:

$$
\begin{aligned}
M_{\alpha+n}(x, I: w) & =\int_{a}^{b}|u-x|^{\alpha+n} w(u) \mathrm{d} u \\
& \leq L_{\alpha+n}(x) \int_{a}^{b} w(u) \mathrm{d} u=L_{\alpha+n}(x) m_{0}(I ; w) .
\end{aligned}
$$

Therefore, from (3.21) we get

$$
\left|\mathcal{O}_{n}(x, f ; w)\right| \leq N \frac{\Gamma(\alpha+1) L_{\alpha+n}(x)}{\Gamma(\alpha+n+1)} .
$$

However, this estimate is worse than one given by (3.23) since obviously

$$
J_{\alpha+n+1}(x)=\frac{1}{b-a} \int_{a}^{b}|u-x|^{\alpha+n} \mathrm{~d} u \leq L_{\alpha+n}(x)
$$


and

$$
\frac{\lambda L_{\alpha+n}(x) J_{\alpha+n+1}(x)}{L_{\alpha+n}(x)-J_{\alpha+n+1}(x)+\lambda J_{\alpha+n+1}(x)} \leq L_{\alpha+n}(x) .
$$

It is easy to see that the inequality in (3.26) can be strict.

Remark 4 Theorem 8 is a generalization of Theorem 3 since for $n=0$ (3.21) and (3.23) reduce to (1.12) and (1.13), respectively.

COROLlary 1 Assume that, for some $n \in \mathbf{N} \cup\{0\}, f^{(n)}(t)$ exists for all $t \in I$. Suppose that $f^{(n)}(\cdot)$ is $N$-Lipschitzian for some constant $N>0$. Then, for all $x \in I$ we have

$$
\left|\mathcal{O}_{n}(x, f ; w)\right| \leq N \frac{M_{n+1}(x, I: w)}{(n+1) ! m_{0}(x, I ; w)}
$$

and

$$
\left|\mathcal{O}_{n}(x, f)\right| \leq N \frac{(x-a)^{n+2}+(b-x)^{n+2}}{(n+2) !(b-a)}
$$

If

$$
0<c \leq w(t) \leq \lambda c, \quad t \in I
$$

for some constants $c$ and $\lambda$, then for all $x \in I$

$$
\left|\mathcal{O}_{n}(x, f ; w)\right| \leq N \frac{\lambda L_{n+1}(x) J_{n+2}(x)}{(n+1) !\left[L_{n+1}(x)-J_{n+2}(x)+\lambda J_{n+2}(x)\right]},
$$

where $L_{n+1}(x)$ and $J_{n+2}(x)$ are defined by (3.24).

Proof Apply Theorem 8 with $\alpha=1$ and note that $\Gamma(k)=(k-1)$ ! for $k \in \mathbf{N}$.

\subsection{Inequalities Involving $f^{(n+1)}$}

The integral identity (2.7) can be used in the similar way as the identity (2.5) in Section 3.1. If $p$ and $q$ are such that (3.8) holds, then from (2.7) 
we get

$$
\begin{aligned}
\left|\mathcal{O}_{n}(x, f ; w)\right| & =\frac{\left|\mathcal{R}_{n}(x, f ; w)\right|}{m_{0}(I ; w)} \\
& \leq \frac{1}{m_{0}(I ; w)} \int_{a}^{b}\left|f^{(n+1)}(t)\right|\left|K_{n+1}(x, t ; w)\right| \mathrm{d} t \\
& \leq \frac{\left\|f^{(n+1)}(\cdot)\right\|_{p}\left\|K_{n}(x, \cdot ; w)\right\|_{q}}{m_{0}(I ; w)} .
\end{aligned}
$$

For $p=1$ and $q=\infty$ from (3.27) we get

$$
\left|\mathcal{O}_{n}(x, f ; w)\right| \leq \frac{\left\|K_{n}(x, \cdot ; w)\right\|_{\infty}}{m_{0}(I ; w)} \int_{a}^{b}\left|f^{(n+1)}(t)\right| \mathrm{d} t
$$

Also, using (3.10) we get

$$
\left|\mathcal{O}_{n}(x, f)\right| \leq \frac{[(b-a) / 2+|x-(a+b) / 2|]^{n+1}}{(n+1) !(b-a)} \int_{a}^{b}\left|f^{(n+1)}(t)\right| \mathrm{d} t
$$

Further, for $1<p, q<\infty$, from (3.27) and (3.11) we get

$$
\left|\mathcal{O}_{n}(x, f)\right| \leq \frac{\left\|f^{(n+1)}(\cdot)\right\|_{p}}{(n+1) !(b-a)}\left[\frac{(x-a)^{q(n+1)+1}+(b-x)^{q(n+1)+1)}}{q(n+1)+1}\right]^{1 / q}
$$

The most interesting case is when $p=\infty$ and $q=1$.

THEOREM 9 Assume that, for some $n \in \mathbf{N} \cup\{0\}, f^{(n+1)}(t)$ exists for all $t \in I$. Suppose that $f^{(n+1)}(\cdot)$ is bounded and integrable on I. Then, for all $x \in I$ we have

$$
\begin{aligned}
\left|\mathcal{O}_{n}(x, f ; w)\right| & \leq \frac{\left\|f^{(n+1)}(\cdot)\right\|_{\infty} M_{n+1}(x, I: w)}{(n+1) ! m_{0}(I ; w)} \\
& \leq \frac{\left\|f^{(n+1)}(\cdot)\right\|_{\infty} L_{n+1}(x)}{(n+1) !}
\end{aligned}
$$


and

$$
\left|\mathcal{O}_{n}(x, f)\right| \leq \frac{\left\|f^{(n+1)}(\cdot)\right\|_{\infty}\left[(x-a)^{n+2}+(b-x)^{n+2}\right]}{(n+2) !(b-a)}
$$

If

$$
0<c \leq w(t) \leq \lambda c, \quad t \in I
$$

for some constants $c$ and $\lambda$, then for all $x \in I$

$$
\left|\mathcal{O}_{n}(x, f ; w)\right| \leq \frac{\left\|f^{(n+1)}(\cdot)\right\|_{\infty} \lambda L_{n+1}(x) J_{n+2}(x)}{(n+1) !\left[L_{n+1}(x)-J_{n+2}(x)+\lambda J_{n+2}(x)\right]}
$$

where $L_{n+1}(x)$ and $J_{n+2}(x)$ are defined by (3.24).

Proof The first inequality in (3.28) follows from (3.27) and (3.12). The second inequality in (3.28) is a consequence of the estimate (see the proof of Theorem 8):

$$
\begin{aligned}
\frac{M_{n+1}(x, I: w)}{m_{0}(I ; w)} & \leq \max \left\{(x-a)^{n+1},(b-x)^{n+1}\right\} \\
& =L_{n+1}(x)
\end{aligned}
$$

The inequality in (3.29) follows from (3.27) and (3.11) with $q=1$. If we put $\alpha=1$ in (3.25), then we get

$$
\frac{M_{n+1}(x, I: w)}{m_{0}(I ; w)} \leq \frac{\lambda L_{n+1}(x) J_{n+2}(x)}{L_{n+1}(x)-J_{n+2}(x)+\lambda J_{n+2}(x)}
$$

and (3.30) follows from (3.28).

Remark 5 The estimate given by (3.30) is better than the estimate

$$
\left|\mathcal{O}_{n}(x, f ; w)\right| \leq \frac{\left\|f^{(n+1)}(\cdot)\right\|_{\infty} L_{n+1}(x)}{(n+1) !}
$$


which follows from the second inequality in (3.28). Namely, (3.26) with $\alpha=1$ gives

$$
\frac{\lambda L_{n+1}(x) J_{n+2}(x)}{L_{n+1}(x)-J_{n+2}(x)+\lambda J_{n+2}(x)} \leq L_{n+1}(x),
$$

and this inequality can be strict.

Remark 6 Note that (3.29) can be rewritten as

$$
\begin{aligned}
& \mid \frac{1}{b-a} \int_{a}^{b} f(t) \mathrm{d} t-f(x) \\
& -\frac{1}{b-a} \sum_{j=1}^{n} \frac{f^{(j)}(x)}{(j+1) !}\left[(b-x)^{j+1}+(-1)^{j}(x-a)^{j+1}\right] \mid \\
& \quad \leq \frac{\left\|f^{(n+1)}\right\|_{\infty}}{(n+2) !(b-a)}\left[(x-a)^{n+2}+(b-x)^{n+2}\right] .
\end{aligned}
$$

This inequality, by the triangle inequality, implies (1.2). However, if we assume $f^{(j)}(x)=0, j=1, \ldots, n$, then (3.29) reduces to (1.3).

Remark 7 Using (1.9) we get

$$
\frac{E_{1}(x, I ; w)}{m_{0}(I ; w)}=\frac{m_{1}(I ; w)-x m_{0}(I ; w)}{m_{0}(I ; w)}=\mu(I ; w)-x
$$

and

$$
\mathcal{O}_{1}(x, f ; w)=\frac{1}{m_{0}(I ; w)} \int_{a}^{b} f(t) w(t) \mathrm{d} t-f(x)-[\mu(I ; w)-x] f^{\prime}(x) .
$$

Also

$$
\begin{aligned}
\frac{M_{2}(x, I ; w)}{m_{0}(I ; w)} & =\frac{E_{2}(x, I ; w)}{m_{0}(I ; w)} \\
& =\frac{m_{2}(I ; w)-2 x m_{1}(I ; w)+x^{2} m_{0}(I ; w)}{m_{0}(I ; w)} \\
& =\frac{m_{2}(I ; w)}{m_{0}(I ; w)}-2 x \mu(I ; w)+x^{2} \\
& =\sigma^{2}(I ; w)+[\mu(I ; w)-x]^{2}
\end{aligned}
$$


and

$$
L_{2}(x)=\left[\frac{b-a}{2}+\left|x-\frac{a+b}{2}\right|\right]^{2}
$$

Now it is easy to check that the first part of Theorem 9 for $n=1$ coincides with Theorem 2. Moreover, as we noted in Remark 5, the estimate given by (3.30) in the case $n=1$ is an improvement of the estimate given by the second inequality in (1.10).

\section{References}

[1] G.A. Anastassiou, Ostrowski type inequalities, Proc. Amer. Math. Soc., 123 (1995), 3775-3781.

[2] S.S. Dragomir, P. Cerone, J. Roumeliotis and S. Wang, A weighted version of Ostrowski inequality for mappings of Hölder type and applications in numerical analysis. RGMIA, 2(1) (1999).

[3] A.M. Fink, Bound on the deviation of a function from its averages, Czechoslavak Math. J., 42(117) (1992), 289-310.

[4] J. Karamata, O prvom stavu srednjih vrednosti odredjenih integrala, Glas srpske kraljevske akademije CLIV, Beograd (1933), 119-144.

[5] G.V. Milovanović, On some integral inequalities, Univ. Beograd Publ. Elektrotehn. Fak. Ser. Mat. Fiz., No. 498-541 (1975), 119-124.

[6] G.V. Milovanović, O nekim funkcionalnim nejednakostima, Univ. Beograd Publ. Elektrotehn. Fak. Ser. Mat. Fiz., No. 599 (1977), 1-59.

[7] G.V. Milovanović and J.E. Pečarić, On generalization of the inequality of A. Ostrowski and some related applications, Univ. Beograd Publ. Elektrotehn. Fak. Ser. Mat. Fiz., No. 544-576.

[8] D.S. Mitrinović, J.E. Pečarić and A.M. Fink, Inequalities Involving Functions and Their Integrals and Derivatives, Kluwer Academic Publishers, Dordrecht, Boston/ London (1991).

[9] A. Ostrowski, Über die Absolutabweichung einer differentiebaren Funktion von ihren Integralmittelwert, Comment. Math. Helv., 10 (1938), 226-227.

[10] C.E.M. Pearce and J. Pečarić, On Anastassiou's generalizations of the Ostrowski inequality and related results, J. Comput. Anal. and Applic. (to appear).

[11] J.E. Pečarić and B. Savić, O novom postupku razvijanja funkcija u red i nekim primjenama, Zbornik radova VA KoV (Beograd) 9 (1983), 171-202.

[12] J. Roumeliotis, P. Cerone and S.S. Dragomir, An Ostrowski type inequality for weighted mappings with bounded second derivatives, RGMIA, 1(1) (1998). 